\title{
Increase in the Heart Rate Variability with Deep Breathing in Diabetic Patients after 12-Month Exercise Training
}

\author{
Bhagyalakshmi Sridhar, ${ }^{1}$ Nagaraja Haleagrahara, ${ }^{2}$ Ramesh Bhat, ${ }^{1}$ \\ Anupama Bangra Kulur, ${ }^{3}$ Sridhar Avabratha ${ }^{4}$ and Prabha Adhikary ${ }^{5}$

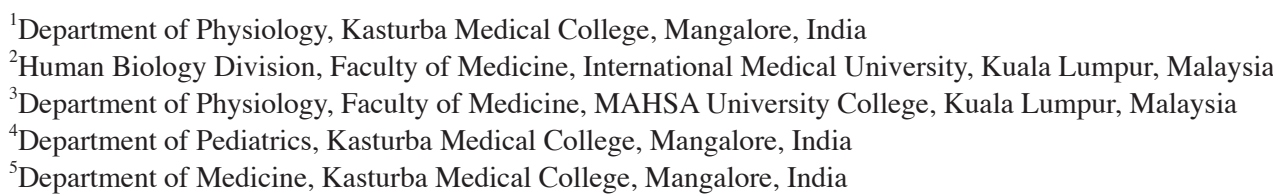

Autonomic neuropathy in diabetes leads to impaired regulation of blood pressure and heart rate variability (HRV), which is due to a shift in cardiac autonomic balance towards sympathetic dominance. Lower HRV has been considered a predictor of cardiac mortality and morbidity. Deep breathing test is a simple method to measure HRV and it provides a sensitive measure of cardiac autonomic function. The effect of long-term physical activity on HRV in type-2 diabetes mellitus is inconclusive. We aimed to evaluate the effects of regular physical exercise on HRV with deep breathing in type 2 diabetes $(n=105)$. Thirty normotensive diabetic patients and 25 hypertensive diabetic patients underwent physical exercise program for 12 months, and the other 50 patients (22 normotensive and 28 hypertensive diabetic patients) were considered the non-exercised group. Electrocardiogram was recorded during deep breathing and HRV was measured. Regular exercise significantly increased HRV in diabetic patients with and without hypertension. The degree of the increase in HRV was greater in hypertensive diabetic patients $(p<0.01)$ than in normotensive diabetic patients $(p<0.05)$. After exercise, glycosylated hemoglobin levels were decreased in both groups of diabetic patients. Moreover, the hypertensive diabetic patients showed a decrease $(p<0.05)$ in blood pressure after regular exercise. Thus, regular exercise training increases HRV, suggesting that there is a shift in the cardiac sympathovagal balance in favor of parasympathetic dominance in diabetic patients. Long-term physical training may be an effective means to reverse the autonomic dysregulation seen in type 2 diabetes.

Keywords: heart rate variability/exercise/diabetes/hypertension/deep breathing

Tohoku J. Exp. Med., 2010, 220 (2), 107-113. (c) 2010 Tohoku University Medical Press

Diabetes mellitus (DM) is a syndrome characterized by chronic hyperglycemia and disturbances of carbohydrate, fat and protein metabolism that are associated with absolute or relative deficiencies in insulin secretion and/or insulin action. The Framingham heart study revealed a marked increase in peripheral artery disease, congestive heart failure, coronary artery disease, and myocardial infarction, as well as a five-fold increase in sudden death in patients with DM (Fox et al. 2007). Diabetes can also cause severe autonomic dysfunction that can be responsible for several disabling symptoms and even sudden cardiac death (American Diabetes Association 1999). The American Heart Association has recently designated $\mathrm{DM}$ as a major risk factor for cardiovascular disease (Powers 2006). Diabetes and hypertension frequently occur concurrently and probably have synergistic detrimental effects on the cardiovascular system. Even in its mildest forms, hypertension is a progressive and lethal disease if left untreated. High blood pressure is also a major risk factor for stroke, coronary heart disease, and renal failure, and the mortality rate in diabetic patients with hypertension is several fold higher than it in normotensive diabetic patients (Takahashi et al. 2001). It is also known that patients with diabetes and hypertension along with obesity, hypercholesterolemia and a sedentary life style are at a greater risk of developing coronary artery disease. In fact, we now know that age, sex, smoking, serum cholesterol, glucose intolerance, exercise and weight may all alter the progression of DM (Takahashi et al. 2001; Fisher and Williams 2005).

Heart rate variability (HRV) refers to the magnitude of the fluctuation in the number of heart beats per minute in conjunction with respiration (Nevruz et al. 2007). HRV has recently become a popular non-invasive research tool in cardiology; in population studies, decreased HRV has been

Received August 3, 2009; revision accepted for publication November 28, 2009. doi:10.1620/tjem.220.107

Correspondence: Nagaraja Haleagrahara, Human Biology Division, Faculty of Medicine and Health, International Medical University

[IMU], 57000 Kuala Lumpur, Malaysia.

e-Mail: hsnagaraja@gmail.com 
of predictive value for mortality among healthy adults (Pomeranz et al. 1985; Tsuji et al. 1994; Kristal-Boneh et al. 1995; Stein and Kleiger 1999). Recent studies have also shown that decreased HRV can indicate an increased risk of arrhythmic events and an increased mortality rate in patients with a previous myocardial infarction (Bigger et al. 1992). Time and frequency domain measures of HRV have provided prognostic information and have also made it possible to perform non-invasive studies on the significance of changes in the regulation of heart rate. Studies have indicated that diabetic patients have a reduced HRV (Wheeler and Watkins 1973; Ewing 1983; Malliani et al. 1991; Liao et al. 2002), while a further study has shown that diabetes together with hypertension can impair cardiac autonomic function (Takahashi et al. 2001). Therefore, HRV can be used in the assessment of the degree of cardiovascular autonomic neuropathy that leads to an increase in mortality in diabetic patients.

One-minute deep breathing-heart rate variability (DBHRV) is a short and simple bedside method for testing autonomic control of the heart (Tamosiunaite et al. 2005; Shuvy et al. 2008). This method has been validated as an independent predictor of cardiac autonomic function in a few cardiac diseases, including myocardial infarction (Tengiz et al. 2002). Deep breathing-induced changes in heart rate occur because of alterations in cardiac parasympathetic activity. When this system is impaired, deep breathing leads to a decrease in HRV (Katona and Jih 1975; Tamosiunaite et al. 2005). The changes in heart rate associated with respiratory activity are mediated by changing levels of vagal and sympathetic activities, as well as by the mechanically induced stretch of the sinus node with each respiration. The degree of the contribution of each of these components is related to the frequency and amplitude of the respiratory signal, the mean level of vagal and sympathetic activity, and the mechanical state of the airways (Van Ravenswaaij-Aris et al. 1993; Katz et al. 1999; Tamosiunaite et al. 2005).

In general, lower HRV has been found to be a significant predictor of cardiac mortality and morbidity, indicating that a high HRV is more desirable (Stein and Kleiger 1999). Pharmacological interventions (Zaliunas et al. 2005), exercise training (Malfatto et al. 1996; Deligiannis et al. 1999; Stein et al. 1999; Carter et al. 2003) and diaphragmatic breathing (Peper and Tibbetts 1992) are known to markedly improve HRV. However, controlled, randomized, long-term studies on the effect of regular physical activity on one-minute DB-HRV are not available. Therefore, we have undertaken this study to examine the effect of regular, physical exercise on DB-HRV in normotensive patients and hypertensive patients with type $2 \mathrm{DM}$.

\section{Materials and Methods}

One hundred and five type 2 DM patients, both men and women, who attended a hospital-based diabetes camp, were randomly chosen for inclusion in this study. All of the subjects gave informed consent, and the study protocol was approved by the Ethics Committee of
Kasturba Medical College. Patients were interviewed and then invited for a baseline clinical examination. A general physical examination, including height, body weight, waist-hip ratio, blood pressure, pulse rate, respiratory rate, and a complete systemic examination were performed. Detailed examinations for the presence of pulmonary vascular disease, cerebral vascular disease, and coronary artery disease were also performed. All measurements were carried out between 9 am and $10 \mathrm{am}$. Subjects were asked to refrain from the intake of tea, coffee, alcohol, or a heavy meal for $4 \mathrm{~h}$ before the physiological assessment. In addition, a 12 lead resting electrocardiogram was recorded, and blood was drawn from the antecubital vein of the patients when seated. Blood samples were used to measure fasting blood glucose (using the glucose dehydrogenase method), and glycosylated hemoglobin $\left(\mathrm{HbA}_{1 \mathrm{C}}\right)$ (by the immunoterbidimetric method). All the subjects were briefed about the protocol and they gave their informed consent to participate in this study. The study complies all the principles postulated in the Declaration of Helsinki in 1983.

DM patients were classified as having diabetes on the basis of history, regardless of the duration of disease or the need for anti-diabetic medications; diabetes was defined as fasting glucose $\geq 7.0$ $\mathrm{mmol} / \mathrm{l}$. DM patients with congestive heart failure, atrial fibrillation, frequent ectopic beats, unstable angina, patients with myocardial infarction, chronic obstructive airway disease, and any patients unable to perform deep breathing were excluded from this study. Diabetes patients without hypertension were defined as patients who had never been treated for hypertension, and had systolic blood pressure $<140$ $\mathrm{mmHg}$ and a diastolic blood pressure $<90 \mathrm{mmHg}$. Diabetes patients with hypertension were defined as patients who had never been treated for hypertension, with a systolic blood pressure $>140 \mathrm{mmHg}$ and/or a diastolic blood pressure $>90 \mathrm{mmHg}$, as well as diabetes patients on antihypertensive drugs. Blood pressure was recorded using a mercury sphygmomanometer in the sitting position after the subjects had relaxed for $20 \mathrm{~min}$. Of the 105 diabetes patients, 55 were randomly chosen for enrollment in the exercise program, which was conducted under the guidance of a physiotherapist. All 55 of these patients were requested to visit the hospital regularly ( 5 days per week) and practice the exercise. Of the 55 type 2 diabetes patients enrolled in the exercise program, 25 patients had hypertension $(\mathrm{DM}+\mathrm{HTN})$ and 30 had no hypertension (DM alone).

\section{Exercise program}

The exercise program included warm up exercises for $5 \mathrm{~min}$, followed by, cycling or treadmill exercise, for $30 \mathrm{~min}$ and cool down exercise for $10 \mathrm{~min}$. The entire duration of the exercise did not exceed $60 \mathrm{~min}$ for each session on each day.

The remaining 50 diabetes patients who were not included in the exercise program were considered the 'no exercise' diabetes group; this group included $28 \mathrm{DM}+\mathrm{HTN}$ patients and $22 \mathrm{DM}$ alone patients. All patients in both groups were examined for DB-HRV. The pharmacological treatment did not differ significantly between the two groups at baseline and there were no major changes in medications during the study.

\section{HRV analysis}

A deep breathing heart rate (HR) test (Katz et al. 1999, Shuvy et al. 2008) was conducted with patients in a supine position during a standard ECG recording. Before beginning the test, subjects were taught to breathe at a rate of 6 respiratory cycles per minute, $5 \mathrm{sec}-$ onds for each inhalation and 5 seconds for each exhalation. The elec- 
trocardiogram was recorded continuously at a speed of $25 \mathrm{~mm} / \mathrm{s}$ for 60 seconds while the patients breathed as instructed. Beat-to-beat alterations in HR were evaluated by the time domain method. The HRV interval (R-R intervals between adjacent QRS complexes resulting from sinus node depolarization) was measured manually with a scaled caliper. The R-R interval was measured in each respiratory cycle and an average R-R interval was taken for the measurement of HRV. The variability in HR was calculated as the difference between the shortest and longest R-R intervals, expressed in beats/min. Each patient in the exercise study group was examined every month on a regular basis.

The study was continued for a period of 12 months. During the follow up, blood pressure, fasting blood glucose, $\mathrm{HbA}_{1 \mathrm{C}}$ levels, body weight, and DB - HRV were measured. To avoid the influences of previous training sessions on HRV, the patients were not allowed to perform physical exercise the day before examination. No changes were made in the medications or lifestyle as reported by the patients.

\section{Statistical analysis}

The results are presented as mean \pm standard deviation (S.D.). The Mann-Whitney $U$ test was employed to compare the demographic and clinical characteristics in the exercised and non exercised groups. The paired Student's $t$ test was employed for statistical comparison between the baseline and follow-up measurements of $\mathrm{HRV}, \mathrm{HbA}_{1 \mathrm{C}}$ and blood pressure. An unpaired Student's $t$ test was used to compare parameters from the DM alone group and the DM + HTN groups, in cases of both exercise and no exercise. A stepwise multiple regression analysis was used for assessing the relationship between variables. The $p$ value was taken as significant at a 5\% confidence level $(p<$ $0.05)$.

\section{Results}

The demographic characteristics of the patients are given in Table 1. Comparisons of the exercised and control groups for various parameters using the Mann-Whitney $U$ test showed that the study groups were well balanced, and that individuals in both groups did not differ significantly in age, gender, duration of diabetes, medication, or smoking status.

The baseline HRV of DM + HTN patients in the exercised group was significantly lower $(p<0.05)$ than the baseline HRV of DM alone patients (Table 2). Regular exercise training for a period of 12 months significantly increased the HRV in both the DM + HTN group $(p<0.01)$ and the DM alone group $(p<0.05)$. However, the increase in the HRV after exercise was greater in the DM + HTN group $(p<0.001)$ compared to the DM alone group $(p<$ 0.01 ), even though the HRV remained significantly lower $(p<0.01)$ in DM + HTN group compared to the DM alone group after 12 months of the exercise program.

There was no statistically significant difference in the $\mathrm{HbA}_{1 \mathrm{C}}$ levels in the DM alone and DM + HTN groups at the baseline recordings (Table 2). After 12 months of the exercise program, the $\mathrm{HbA}_{1 \mathrm{C}}$ levels were decreased significantly in the DM alone group $(p<0.001)$ and in the DM+HTN group $(p<0.01)$. However, the decrease in the $\mathrm{HbA}_{1 \mathrm{C}}$ level was significantly greater $(p<0.01)$ in the DM alone group than in the DM + HTN group (Table 2).

Even in the non-exercised group, the baseline HRV of the DM + HTN patients was significantly lower $(p<0.01)$ than in the DM alone patients (Table 3). After 12 months, the HRV of both these DM + HTN patients and DM alone patients did not show any significant change when compared to baseline. However, the HRV of the DM + HTN group was significantly lower $(p<0.05)$ than the HRV of the DM alone group; even at 12 months follow up. Thus when HRV was compared after 12 months between the exercised and non-exercised groups in both DM alone patients and DM + HTN patients, a significant increase was seen only in the DM alone patients from the exercised group. A similar change was also seen in exercised DM +

Table 1. Demographic and clinical characteristics of the study group.

\begin{tabular}{lccc}
\hline \multicolumn{1}{c}{ Parameters } & $\begin{array}{c}\text { Non-exercised group } \\
(50)\end{array}$ & $\begin{array}{c}\text { Exercised group } \\
(55)\end{array}$ & $p<$ \\
\hline Age (years) (mean \pm S.D.) & $59.45 \pm 2.75$ & $61.78 \pm 3.10$ & 0.419 \\
Gender (M/F) & $28 / 22$ & $30 / 25$ & 0.092 \\
BMI (kg/m $\left.{ }^{2}\right)$ & $27.01 \pm 0.22$ & $27.82 \pm 0.19$ & 0.321 \\
Duration of diabetes (yrs) & $7.00 \pm 1.44$ & $8.89 \pm 1.28$ & 0.063 \\
(mean \pm s.D.) & & & \\
Diabetes Treatment (\%) & 3 & 6 & 0.716 \\
$\quad$ Insulin & 13 & 19 & 1.00 \\
$\quad$ Sulphonylureas & 3 & 4 & 0.351 \\
$\quad$ Other drugs & 4 & 6 & 0.905 \\
$\quad$ Smoking (\%) & 8 & & \\
Hypertension Treatment & 11 & 6 & 0.812 \\
$\quad$ ACE inhibitors & 4 & 8 & 0.094 \\
$\quad$ Beta blockers & 1 & 5 & 0.649 \\
Calcium channel blockers & & 2 & 0.668 \\
\hline Diuretics & & & \\
\hline
\end{tabular}


Table 2. HRV and $\mathrm{HbA}_{1 \mathrm{C}}$ levels (mean \pm S.D.) before and after an exercise program.

\begin{tabular}{clcc}
\hline & Parameters & DM & DM + HTN \\
\hline HRV & Baseline & $13.27 \pm 1.91$ & $12.24 \pm 0.82^{*}$ \\
(beats/min) & After 12 months & $17.73 \pm 0.94 *$ & $15.71 \pm 0.61^{* *}$ \\
$\mathrm{HbA}_{1 \mathrm{C}}$ & Baseline & $8.61 \pm 0.74$ & $8.50 \pm 0.38^{\mathrm{NS}}$ \\
$(\%)$ & After 12 months & $6.40 \pm 0.45^{* * *}$ & $7.44 \pm 0.44 * *$ \\
\hline
\end{tabular}

$* p<0.05 ; * * p<0.01 ; * * * p<0.001$; DM, diabetes mellitus; HRV, heart rate

variability; HTN, hypertension; NS, not significant.

Table 3. Follow-up HRV and $\mathrm{HbA}_{1 \mathrm{C}}$ levels (mean \pm S.D.) in the non-exercised group.

\begin{tabular}{clcc}
\hline & Parameters & DM & DM + HTN \\
\hline HRV & Baseline & $14.48 \pm 0.73$ & $12.19 \pm 0.64^{* *}$ \\
(beats/min) & After 12 months & $14.12 \pm 0.65^{\mathrm{NS}}$ & $13.02 \pm 0.54^{*} \mathrm{NS}$ \\
$\mathrm{HbA}_{1 \mathrm{C}}$ & Baseline & $8.61 \pm 0.63$ & $8.7 \pm 0.32$ \\
$(\%)$ & After 12 months & $9.59 \pm 0.44^{\mathrm{NS}}$ & $9.84 \pm 0.53^{\mathrm{NS}}$ \\
\hline
\end{tabular}

$* p<0.05 ; * * p<0.01$

DM, diabetes mellitus; HRV, heart rate variability; HTN, hypertension; NS, Not significant.

Table 4. HRV between exercised and non-exercised groups after 12 months.

\begin{tabular}{|c|c|c|c|c|}
\hline \multirow{2}{*}{ Parameter } & \multicolumn{2}{|c|}{$\mathrm{DM}$} & \multicolumn{2}{|c|}{$\mathrm{DM}+\mathrm{HTN}$} \\
\hline & Exercised & Non-exercised & Exercised & Non-exercised \\
\hline HRV (beats/min) & $17.73 \pm 0.94 * *$ & $14.12 \pm 0.65$ & $15.71 \pm 0.61 *$ & $13.02 \pm 0.57$ \\
\hline
\end{tabular}

Table 5. Changes in blood pressure after 12 months in exercised and non-exercised group.

\begin{tabular}{cccc}
\hline Group & Blood pressure & Baseline $(\mathrm{mmHg})$ & After 12 months $(\mathrm{mmHg})$ \\
\hline \multirow{2}{*}{ Exercised DM + HTN } & Systolic & $144.24 \pm 2.87$ & $135.53 \pm 3.54^{*}$ \\
& Diastolic & $88.59 \pm 3.92$ & $82.82 \pm 1.07^{*}$ \\
Non-exercised DM + HTN & Systolic & $145.22 \pm 3.27$ & $146.03 \pm 4.28^{\mathrm{NS}}$ \\
& Diastolic & $87.07 \pm 3.92$ & $88.15 \pm 3.68^{\mathrm{NS}}$ \\
\hline
\end{tabular}

$* p<0.01$

DM, diabetes mellitus; HRV, heart rate variability; HTN, hypertension; NS, Not significant.

HTN patients (Table 4).

Both systolic and diastolic blood pressure was significantly decreased $(p<0.05)$ in the DM + HTN group after 12 months of regular exercise when compared with baseline levels (Table 5). In the non-exercised DM + HTN group, there was no significant change in blood pressure after 12 months. When the reproducibility measurements for deep breathing heart rate variability was tested, the coefficient of variation $(\mathrm{CV})$ obtained was 6.2 , which indicates that deep breathing HRV had good reproducibility. In the stepwise regression analyses, exercise accounted for $70.2 \%$ of the variation in HRV after 12 months. When other predictors were also included, the largest ' $\mathrm{t}$ ' value was for exercise ( $t=$
5.402; $p<0.001)$, which indicated that exercise has contributed maximally for the observed changes in DB-HRV. A greater co-efficient value for the exercise confirmed that exercise has maximum contributions among all the predictors (age, duration of the diabetes and medications).

\section{Discussion}

Heart rate variability with deep breathing is the simplest and most widely performed measure of autonomic control of the heart. This test produces a sensitive, specific, and reproducible indirect measure of vagal cardiac function (Katz et al. 1999; Bhagyalakshmi et al. 2007). In this study, the baseline HRV in DM + HTN patients was significantly 
lower than the baseline HRV of DM alone patients, in both exercised and non exercised groups. This indicates that cardiac autonomic functions had been negatively affected in patients having diabetes associated with hypertension. This agrees with a previous study by Takahashi et al. (2001) which demonstrated that hypertension with diabetes will impair cardiac autonomic function.

We found that both DM alone and DM + HTN patients in the exercised group showed a significant improvement in mean DB-HRV with regular, supervised, integrated exercise for a period of 12 months. This indicates that both groups experienced beneficial effects on cardiac autonomic regulation due to regular exercise. This is in line with previous longitudinal studies which have shown that exercise training increases HRV in various conditions, including coronary artery disease (Iellamo et al. 2000), acute myocardial infarction (Malfatto et al. 1996), cardiac rehabilitation patients (Tygesen et al. 2001), in patients on hemodialysis (Deligiannis et al. 1999) and in healthy young or older adults (Stein et al. 1999). Contrary to this, a recent study by Scott et al. (2004) showed that cardiac parasympathetic activity was not enhanced with endurance training. A study by Loimaala et al. (2003) reported a trend towards increasing values of HRV with exercise, and they also observed that resting heart rate decreased significantly with exercise in the study group, which suggests that vagal tone was increased after training. Our results agree with those of Loimaala et al. (2003), and in addition, we have taken their study further, by using DM + HTN to assess HRV, and by also examining the effects of exercise on $\mathrm{HbA}_{1 \mathrm{C}}$ levels and blood pressure in these patients.

In our study, the increase in HRV after regular exercise was greater in the DM + HTN group than the DM alone group, but the HRV was still remained significantly lower in $\mathrm{DM}+\mathrm{HTN}$ group compared to DM alone group, even after 12 months of the regular exercise program. This indicates that even though both groups experienced beneficial effects on cardiac autonomic function from regular exercise, the beneficial effect was greater in the DM + HTN group. This may be due to both the increased blood sugar and the increased blood pressure in diabetes associated with hypertension, since regular exercise is known to reduce both blood sugar and blood pressure (Malfatto et al. 1996; Iellamo et al. 2000). Analysis of HRV in the non-exercised group (both DM alone and DM + HTN patients) indicated that there was no statistically significant improvement in HRV in either group after 12 months; in fact there was actually a decreasing trend in mean HRV, which might be due to the natural course of the disease which affects cardiac autonomic function and also due to the ageing process (Deligiannis et al. 1999; Stein et al. 1999; Oka et al. 2002).

In this study, in exercised patients, both the DM alone and DM + HTN groups showed a significant reduction in mean $\mathrm{HbA}_{1 \mathrm{C}}$ levels with regular, exercise training for a period of 12 months. However, the reduction in the $\mathrm{HbA}_{1 \mathrm{C}}$ levels was greater in the DM alone group than in the DM +
HTN group. This indicates that both groups experienced a beneficial effect on glycemic control by regular exercise, but this effect was more pronounced in the DM alone group. In the non-exercised group, both DM alone and DM + HTN patients did not show any significant change in $\mathrm{HbA}_{1 \mathrm{C}}$ levels after 12 months. The decrease in $\mathrm{HbA}_{1 \mathrm{C}}$ level after 12 months of exercise is in agreement with previous studies by Loimaala et al. (2003) and Canche et al. (2005), which showed a similar significant decrease in $\mathrm{HbA}_{1 \mathrm{C}}$ levels with exercise training in type $2 \mathrm{DM}$ patients. This confirms that regular, physical exercise in diabetes patients improves blood glucose homeostasis and diabetes control.

In the present study, a significant reduction in both systolic and diastolic blood pressure was observed in the DM + HTN group after 12 months regular exercise. Again, this is in agreement with previous studies (Hagberg et al. 1989; Gordon et al. 1997; Bacon et al. 2004), which showed that both mean systolic blood pressure and mean diastolic blood pressure were reduced in hypertensive patients with exercise training.

Chronic hyperglycemia is an important factor that leads to various complications of DM. Large, randomized clinical trials of subjects with type 2 DM have conclusively demonstrated that a reduction in chronic hyperglycemia prevents or delays macrovascular and microvascular complications such as neuropathy, nephropathy and retinopathy. Skeletal muscles are the major site for metabolic fuel consumption in the resting state, and the increased muscle activity during vigorous aerobic exercise greatly increases fuel requirements. For diabetes individuals, exercise is useful both for lowering plasma glucose levels during and following exercise, and for increasing insulin sensitivity. Therefore, regular exercise training is effective in the reduction of hyperglycemia and also has multiple positive benefits including cardiovascular risk reduction.

Absence of metabolic data and a lack of accompanying cardiovascular measures, such as the effect of different meal compositions on HRV, measurement of exercise capacity (peak $\mathrm{VO}_{2}$ ), as well as the absence of echocardiographic data, are the major limitations of our study. One of the major difficulties we faced is the lack of frequency domain HRV analysis, which is the more widely accepted method of HRV analysis and which has higher sensitivity and specificity. However, the clinical usefulness of DB-HRV measurements from short term ECG recordings is well established. However, the influence of drug treatment and its discontinuation on HRV also needs to be carefully considered.

In conclusion, this study has shown that regular, exercise training significantly improves DB-HRV and also reduces $\mathrm{HbA}_{1 \mathrm{C}}$ in both type $2 \mathrm{DM}$ patients with and without hypertension, and also reduces the blood pressure in diabetes with hypertension. However, the improvement in HRV was greater in diabetes patients with hypertension, while the reduction in $\mathrm{HbA}_{1 \mathrm{C}}$ was greater in diabetes patients without hypertension. Regular, physical exercise may have benefi- 
cial effects on the autonomic control of the heart, and these effects may have clinical importance in preventing sudden cardiac death in diabetes. This study is one of the few studies on the effect of a supervised exercise program on DBHRV in DM patients. More epidemiological data and a larger sample of patients are needed to confirm that the beneficial effects observed in these autonomic and metabolic variables will ultimately have favorable effects on the clinical outcomes in diabetic patients.

\section{References}

American Diabetes Association. (1999) Diabetes Mellitus: A major risk factor for cardiovascular disease. A joint editorial statement by the American Diabetes Association; the National Heart, Lung, and Blood Institute; the Juvenile Diabetes Foundation International; the National Institute of Diabetes and Digestive and Kidney Diseases; and the American Heart Association. Circulation, 100, 1132-1133.

Bacon, S.L., Sherwood, A., Hinderliter, A. \& Blumenthal, J.A. (2004) Effects of exercise, diet and weight loss on high blood pressure. Sports Med., 34, 307-316.

Bhagyalakshmi, S., Nagaraja, H.S., Anupama, B.K., Bhat, R., Adhikari, P., Murthy, N. \& Avabratha, S. (2007) Effect of supervised integrated exercise on heart rate variability in type 2 diabetes mellitus. Kardiol. Pol., 65, 363-368.

Bigger, J.T., Fleiss, J.L., Steinman, R.C., Rolnitzky, L.M., Kleiger, R.E. \& Rottman, J.N. (1992) Frequency domain measures of heart period variability and mortality after myocardial infarction. Circulation, 85, 164-171.

Canche, M.K.A. \& Gonzalez, S.B.C. (2005) Endurance training in adults with diabetes mellitus type 2. Rev. Lat. Am. Enfermagem, 13, 21-26.

Carter, J.B., Banister, E.W. \& Blaber, A.P. (2003) The effect of age and gender on heart rate variability after endurance training. Med. Sci. Sports Exerc., 35, 1333-1340.

Deligiannis, A., Kouidi, E. \& Tourkantonis, A. (1999) Effects of physical training on heart rate variability in patients on haemodialysis. Am. J. Cardiol., 84, 197-202.

Ewing, D.J. (1983) Practical bedside investigation of diabetic autonomic failure. In: Bannister, S.R. Autonomic failure a text book of clinical disorders of the autonomic nervous system. Oxford University Press, 371-403.

Fisher, N.D.L. \& Williams, G.H. (2005) Hypertensive vascular disease. In: Kasper D.L., Braunwald, E., Fauci, A.S., Hauser, S.L., Longo, D.L. \& Jameson, J.L., eds., Harrison's Principles of Internal Medicine $16^{\text {th }}$ edition., The Mc Graw Hill companies Inc. New York, 1463-1480.

Fox, C.S., Coady, S., Sorlie, P.D., D’Agostino, R.B., Pencina, M.J., Vasan, R.S., Meigs, J.B., Levy, D. \& Savage, P.J. (2007) Increasing cardiovascular disease burden due to diabetes mellitus: The Framingham Heart Study. Circulation, 115, 15441550.

Gordon, N.F., Scott, C.B. \& Levine, B.D. (1997) Comparison of single versus multiple lifestyle interventions: are the antihypertensive effects of exercise training and diet-induced weight loss additive? Am. J. Cardiol., 79, 763-767.

Hagberg, J.M., Montain, S.J., Martin, W.H. \& Ehsani, A.A. (1989) Effect of exercise training in 60- to 69-year old persons with essential hypertension. Am. J. Cardiol., 64, 348-353.

Iellamo, F., Legramante, J.M., Massaro, M., Raimondi, G. \& Galante, A. (2000) Effects of residential exercise training on baroreflex sensitivity and heart rate variability in patients with coronary artery disease: A randomized, controlled study. Circulation, 102, 2588-2592.

Katona, P.G. \& Jih, F. (1975) Respiratory sinus arrhythmia: noninvasive measure of parasympathetic cardiac control. J. Appl.
Physiol., 39, 801-805.

Katz, A., Liberty, I.F., Porath, A., Ovsyscher, I. \& Prystowsky, E.N. (1999) A simple bedside test of 1-minute heart rate variability during deep breathing as a prognostic index after myocardial infarction. Am. Heart J., 138, 32-38.

Kristal-Boneh, E., Raifel, M., Froom, P. \& Ribak, J. (1995) Heart rate variability in health and disease. Scand. J. Work Environ. Health, 21, 85-95.

Liao, D., Carnethon, M., Evans, G.W., Cascio, W.E. \& Heiss, G. (2002) Lower heart rate variability is associated with development of coronary heart disease in individuals with diabetes: the atherosclerosis risk in communities (ARIC) study. Diabetes, 51, 3524-3531.

Loimaala, A., Huikuri, H.V., Kööbi, T., Rinne, M., Nenonen, A. \& Vuori, I. (2003) Exercise training, improves baroreflex sensitivity in type 2 diabetes. Diabetes, 52, 1837-1842.

Malfatto, G., Facchini, M., Bragato, R., Branzi, G., Sala, L. \& Leonetti, G. (1996) Short and long-term effects of exercise training on tonic autonomic modulation of heart rate variability after myocardial infarction. Eur. Heart J., 17, 532-538.

Malliani, A., Pagani, M., Lombardi, F. \& Cerutti, S. (1991) Cardiovascular neural regulation explored in frequency domain. Circulation, 84, 482-492.

Nevruz, O., Yokusoglu, M., Uzun, M., Demirkol, S., Avcu, F., Baysan, O., Koz, C., Cetin, T., Sag, C., Ugur, A. \& Isik, E. (2007) Cardiac autonomic functions are altered in patients with acute leukemia, assessed by heart rate variability. Tohoku J. Exp. Med., 211, 121-126.

Oka, T., Matsukura, M., Okamoto, M., Harada, N., Kitano, T., Miike, T. \& Futatsuka, M. (2002) Autonomic nervous functions in fetal type Minamata disease patients: assessment of heart rate variability. Tohoku J. Exp. Med., 198, 215-221.

Peper, E. \& Tibbetts, V. (1992) Fifteen-month follow-up with asthmatics utilizing EMG/incentive inspirometer feedback. Biofeedback Self Regul., 17, 143-151.

Pomeranz, B., Macauley, R.J., Caudill, M.A., Kutz, I., Adam, D., Gordon, D., Kilborn, K.M., Barger, A.C., Shannon, D.C., Cohen, R.J. et al. (1985) Assessment of autonomic function in humans by heart rate spectral analysis. Am. J. Physiol., 248 , H151-H153.

Powers, A.C. (2006) Diabetes mellitus. In: James, L.J. \& Harrison T.R., (ed). Harrison's Endocrinology. Mc Graw-Hill Professional, New York, 283-332.

Scott, A.S., Eberhard, A., Ofir, D., Benchetrit, G., Dinh, T.P., Calabrese, P., Lesiuk, V. \& Perrault, H. (2004) Enhanced cardiac vagal efferent activity does not explain training-induced bradycardia. Auton. Neurosci., 112, 60-68.

Shuvy, M., Arbelle, J.E., Grosbard, A. \& Katz, A. (2008) A simple test of one minute heart rate variability during deep breathing for evaluation of sympathovagal imbalance in hyperthyroidism. Isr. Med. Assoc. J., 10, 603-606.

Stein, P.K., Ehsani, A., Domitrovich, P.P., Kleiger, R.E. \& Rottman, J.N. (1999) Effect of exercise training on heart rate variability in healthy older adults. Am. Heart J., 138, 567-576.

Stein, P.K. \& Kleiger, R.E. (1999) Insights from the study of heart rate variability. Annu. Rev. Med., 50, 249-261.

Takahashi, N., Nakagawa, M., Saikawa, T., Ooie, T., Yufu, K., Shigematsu, S., Hara, M., Sakino, H., Katsuragi, I., Okeda, T., Yoshimatsu, H. \& Sakata, T. (2001) Effect of essential hypertension on cardiac autonomic function in type 2 diabetic patients. J. Am. Coll. Cardiol., 38, 232-237.

Tamosiunaite, M., Urbonaviciene, G., Vainoras, A., Gargasas, G., Kaminskiene, S., Bluzaite, I. \& Rickli, H. (2005) Influence of deep breathing on heart rate variability in patients with ischemic heart disease. Elektronika Elektrotechnika, 59, 33-36.

Tengiz, I., Ercan, E., Yakut, N., Gurbuz, A. \& Nalbantgil, I. (2002) Hyper acute myocardial infarction and one minute heart rate variability test. Turk. Kardiyol. Dern. Ars., 30, 616-620.

Tsuji, H., Venditti, F.J., Manders, E.S., Evans, J.C., Larson, M.G., 
Feldman, C.L. \& Levy, D. (1994) Reduced heart rate variability and mortality risk in an elderly cohort. The Framingham Heart Study. Circulation, 90, 878-883.

Tygesen, H., Wettervik, C. \& Wennerblom, B. (2001) Intensive home-based exercise training in cardiac rehabilitation increases exercise capacity and heart rate variability. Int. J. Cardiol., 79, $175-182$.

Van Ravenswaaij-Aris, C.M., Kollee, L.A., Hopman, J.C., Stoelinga, G.B. \& Van Geijn, H.P. (1993) Heart rate variabili- ty. Ann. Intern. Med., 118, 436-447.

Wheeler, T. \& Watkins, P.J. (1973) Cardiac denervation in diabetes. Br. Med. J., 4, 584-586.

Zaliunas, R., Brazdzionytc, J., Zabiela, V. \& Jurkevicius, R. (2005) Effects of amlodipine and lacidipine on heart rate variability in hypertensive patients with stable angina pectoris and isolated left ventricular diastolic dysfunction. Int. J. Cardiol., 101, 347-353. 